\title{
Coherence Compilation: Applying AIED Techniques to the Reuse of Educational TV Resources
}

\author{
Rosemary Luckin, Joshua Underwood, Benedict du Boulay, Joe Holmberg and \\ Hilary Tunley \\ IDEAs Laboratory, Human Centred Technology Research group, \\ School of Science and Technology \\ University of Sussex \\ Brighton BN1 9Q UK \\ Email: rosel@sussex.ac.uk
}

\begin{abstract}
The HomeWork project is building an exemplar system to provide individualised experiences for individual and groups of children aged 6-7 years, their parents, teachers and classmates at school. It employs an existing set of broadcast video media and associated resources that tackle both numeracy and literacy at Key Stage 1. The system employs a learner model and a pedagogical model to identify what resource is best used with an individual child or group of children collaboratively at a particular learning point and at a particular location. The Coherence Compiler is that component of the system which is designed to impose an overall narrative coherence on the materials that any particular child is exposed to. This paper presents a high level vision of the design of the Coherence Compiler and sets its design within the overall framework of the HomeWork project and its learner and pedagogical models.
\end{abstract}

\section{Introduction}

The use of TV (and radio) in education has a long history - longer than the use of computers in education. But the traditions within which TV operates, such as the strong focus on narrative and the emphasis on viewer engagement, are rather different from those within which computers in education, and more particularly ITS \& AIED systems operate. We can characterise ITS \& AIED systems as being fundamentally concerned with individualising the experience of learners and groups of learners and supporting a range of representations and reifications of either the domain being explored or the learning process. The traditional division of the subject into student modelling, domain modelling, modelling teaching and interface issues reflects this concern with producing systems that react intelligently to the learner or group of learners using the system. Even where the system is simply a tool or a vehicle to promote collaboration (say), there will be a concern to monitor and perhaps adjust the parameters within which that collaboration takes place, if the system is to be regarded as of interest to the ITS \& AIED community. One novel aspect of the HomeWork system is its concern with modelling and managing the narrative flow of the learners' experience both at the micro level within sessions and at the macro level between sessions and over extended use. This project is building an exemplar system for 
children aged 6-7 years, their parents, teachers and classmates at school to tackle both numeracy and literacy at Key Stage 1. In the classroom the child will be able to work alone or as part of a group and interact both with a large interactive whiteboard and with a handheld digital slate as directed by the teacher. When the handheld slate is taken home further activities can be completed using a home TV and the slate either working alone, with their family, or with other classmates who may be co-located or at a distance in their own homes.

This paper concentrates on the narrative aspects of the HomeWork project and on the Coherence Compiler that ensures narrative coherence. We start by outlining the HomeWork project. We then give the theoretical background to the narrative work. Finally we discuss how the coherence compiler is being designed to maintain narrative coherence across different technologies in different locations despite the interactive interventions of the learners.

\section{The HomeWork Project}

Most homes and schools possess a TV, and many schools now also possess interactive whiteboards. TV is a technology that has been used to deliver motivating, gripping and captivating content to millions of learners of all ages. The introduction of digital interactive broadband systems that can carry information both to and from the user opens up the possibility of personalised, adaptive learning experiences. However, learners are not yet used to interacting through their TV screens, which are not appropriate when it comes to text and navigation. What is required is a learning experience designed for delivery across multiple technologies and interfaces in which the educational media are integrated into a coherent, non-linear narrative and experienced by the learner through the technological artefact that best delivers the media being used. In this way the rich television media can be viewed through the TV interface and the text and associated interactive components through the PC (slate). Our previous work has shown that young children can co-ordinate the integration of multiple interfaces and artefacts [1].

The design challenge is how to string together bits of content (potentially from different providers) across a variety of devices (TV, tablets, paper) and locations (school and home) in such a way that enables learners to engage with the concepts of the discipline being studied (not the technology being employed or the effort of mentally linking the episodes), and to collaborate within and across locations. This requires the development of an underpinning pedagogical framework. To be effective, this framework needs to be grounded in a pedagogy that recognises that education is interactive with a multiplicity of potential participants both human and artefact. It also needs to be flexible enough to apply to a range of devices (both technological and non-technological), real educational contexts, constantly changing policy, and to the evolving future.

In [2] we identified potential points of contact between Social Constructivism and broadband learning, and proposed key actions that provide a starting point for a future design framework. In this framework we expanded the definition of Broadband to describe a concept that accommodates a wide 'bandwidth' of participants, senses, devices and contexts. This Broadband Learner Modelling (BLM) approach expands 
the theoretical framework previously developed for the design of a single user Interactive Learning Environment [3,4] and a Multimedia Interactive CD-ROM [5].

Within the BLM framework a pivotal role is played by the learner-modelling component that is used to profile each learner or group of learners and teachers. This component allows a dynamic description of the learner/s to be maintained and used to shape their interactions and collaborations. The design of the learner model is also used as a template for the design of the descriptive tags that are used to organise the educational resources at the system's disposal. These resources include multiple media (such as text, audio and video) about particular areas of the curriculum (primary maths and literacy in the instance of this grant application) as well as other learners and teachers who can offer collaborative support.

\section{The Learner Model}

Within different contexts such as school and home there are models of learners in the minds of teachers, parents, peers and the learners themselves. These are not linked, but in sum they tell a story of a learner's intellectual development. Through the creation and maintenance of the Broadband Learner Model these different perspectives are brought together as different participants are able to access and update their view of the learner. The minimum core components in the Learner Model have been specified through the ieTV pilot system developed by the authors [2].

This system matches learners, both as individuals and as groups, to the best available learning resources described in its database. This database contains information about multiple media including video, audio and text as well as profiles of other learners and teachers who may be able to offer assistance. The HomeWork system expands this learner model for use across multiple contexts and devices: the learners' slates for use in class and at home, teacher workstation, large screen classroom TV or interactive whiteboard and home TV screen and set-top box. Learners will be able to access an up-to-date representation of themselves in the shape of the Learner Model between the home and school learning contexts via their slates.

\section{An Example Scenario}

The scenario presented in Table 1 below describes the desired learner experience and the proposed system behaviour. 
Table 1. Sample Scenario of Use and associated system behaviour

\begin{tabular}{|c|c|}
\hline Sample Scenario & System Behaviour \\
\hline $\begin{array}{l}\text { Mary is } 6 \text { years old and her school is } \\
\text { tuned into the 'Number Crew' from } \\
\text { Channel 4. The programme and } \\
\text { associated interactive materials are } \\
\text { broadcast to the class's set-top box and } \\
\text { the children watch Bradley and the crew } \\
\text { solve number problems on the good ship } \\
\text { Mathematical. Whilst the TV pictures on } \\
\text { the large classroom screen engage the } \\
\text { class, interactive exercises and activity } \\
\text { sheets are transferred from the set-top } \\
\text { box to each child's 'digital slate'. These } \\
\text { exercises are differentiated and } \\
\text { personalised to each child through the } \\
\text { Broadband Learner Model maintained } \\
\text { by the system for each child. The } \\
\text { Coherence Compiler ensures the } \\
\text { relationship between these exercises and } \\
\text { the opening TV episode are clear }\end{array}$ & $\begin{array}{l}\text { Registration details for Mary and her } \\
\text { classmates have already been used by } \\
\text { the system in collaboration with the } \\
\text { teacher to construct initial learner } \\
\text { profiles. At the start of today's } \\
\text { session the teacher has entered the } \\
\text { lesson parameters (e.g. length) for } \\
\text { which she would like TV programmes } \\
\text { and associated interactive activities. } \\
\text { The BLM coherence compiler } \\
\text { matches the details of the class profile } \\
\text { and the individual learner profiles to } \\
\text { the most appropriate resources in the } \\
\text { BLM content database. These are } \\
\text { offered to the teacher who makes the } \\
\text { final selection for class session and for } \\
\text { the individual learners. Once these } \\
\text { selections are made, the TV material is } \\
\text { displayed on the classroom large } \\
\text { screen. The teacher workstation and } \\
\text { the children's slates are synchronized } \\
\text { and the learner profiles updated to } \\
\text { reflect the activities completed. }\end{array}$ \\
\hline $\begin{array}{l}\text { When Bradley and the crew say goodbye } \\
\text { each child's slate comes to life and the } \\
\text { children can work as individuals (or in } \\
\text { pairs or small groups) with the } \\
\text { interactive materials on the Number } \\
\text { Crew website in the Channel } 4 \\
\text { Collaborative Learning Community. } \\
\text { Mary and Jo, her classmate, work } \\
\text { together. }\end{array}$ & $\begin{array}{l}\text { After (and possibly during) the TV } \\
\text { programme the children complete the } \\
\text { interactive activities selected for them. } \\
\text { The Learner model for each child is } \\
\text { updated on the slate as they progress } \\
\text { and subsequently synchronised with } \\
\text { the teacher's workstation. At the end } \\
\text { of the lesson the teacher can select the } \\
\text { material for the child to take home or. }\end{array}$ \\
\hline $\begin{array}{l}\text { That night at home, Mary switches on } \\
\text { her slate and it automatically } \\
\text { synchronises with the home set-top box. } \\
\text { The Coherence Compiler provides her } \\
\text { with a televisual summary of her recent } \\
\text { study leading to Mary's teacher's } \\
\text { suggestion that some consolidation on } \\
\text { addition would be good. When Mary's } \\
\text { mum turns on the TV, Bradley engages } \\
\text { Mary in some number puzzles that she } \\
\text { then completes on her slate with some } \\
\text { help from classmate Jo, who lives on the } \\
\text { other side of town. }\end{array}$ & $\begin{array}{l}\text { At home the Leaner model on the slate } \\
\text { is synchronised with that stored on, or } \\
\text { delivered to the TV, set top box so that } \\
\text { when the slate is turned on the } \\
\text { relevant TV programme material can } \\
\text { be played. Mary can contact Jo, who } \\
\text { has the same set up at his house and } \\
\text { they can once again work together for } \\
\text { a while. Both Mary and Jo's Leaner } \\
\text { model is updated by the system. } \\
\text { When they go to school the next day } \\
\text { their slates are synchronised with that } \\
\text { of the teacher workstation. }\end{array}$ \\
\hline
\end{tabular}




\section{Coherence Compilation}

The Coherence compiler is an attempt to operationalise guidelines drawn from the Non-linear Interactive Narrative Framework. The original Non-linear Interactive Narrative Framework (NINF) was the product of the MENO research project [6]. This framework was subsequently adapted and used in the design of the IETV pilot system developed at Sussex [2] and is now being further expanded for use in the HomeWork project. In this section of the document we discuss the relevant theoretical grounding for the NINF, and the influence of previous work, in particular that of the MENO project on the NINF. We then present the current version of the NINF for use in the HomeWork Project.

\subsection{Theoretical Grounding}

[7] describes narrative as "a mode of thought and an expression of a culture's worldview". He suggests that we make sense of our own thoughts and experiences, and those of others through the active generation of narrative. In this sense, narrative shapes our knowledge and experience and is central to our cognition. A narrative can take the form of a story that entices us through a sequence of events. The narrative process allows us to make sense of the world and to share this with others. Narrative can also be used as a framework within which explorations can occur, a macrostructure with a network of causal links and signposts [6]. Within this overarching structure there are inter-related elements each with their own micro-narrative. In fact, within formal education there may be several layers of this structure with a macronarrative that is, for example, at the level of a lesson within which there are different elements. This lesson is itself also part of a term's curriculum and therefore in a sense a micro-narrative too. From the point of view of learning and the HomeWork project in particular, we need to offer a means of helping teachers and learners see the links between the layers of macro and micro narratives as well as to keep track of the individual narrative elements themselves. This is what we refer to as Narrative Guidance. This guidance needs to be adaptive to the needs of the learner/s, it needs to offer a strong 'storyline' when a learner is new to a subject and then fade as he becomes more accomplished. The important factor here is that the learner/s must participate in the activity of creating the links between the elements of the narrative.

Social Constructivism [8] has been influential within mainstream education and the design of educational technology alike for the latter part of the twentieth century. It requires that both learners and teachers are active participants in a process of mediated communication. So what does all this have to do with the role of interactive technology? The point about interactive technology is that it allows us to "play around' with the nature of the narrative guidance we offer to a learner, it allows the learner to be more active in the path they take (or create) through the resources and experiences they are offered. The problem that can arise is that learners have too much freedom to explore and end up being confused and lost. There is a fluctuating tension between the strength of the guidance we need to offer and the amount of control we leave with the learner. We need to provide them with tools to help them construct their own understanding from their experiences. We also need to free learners to explore their own curiosity and to be creative. 
It is this need to support learner creativity that provides us with a third theoretical position to explore. Creativity can be considered as a process through which individuals, groups and even entire societies are able to transcend an accepted model of reality. It has been differentiated by [9] into three broad categories: combinatorial, exploratory and transformational all of which require the manipulation of an accepted familiarity, pattern or structure in order to produce a novel outcome. The perceptions of reality that are the backdrop for creativity vary not only from individual to individual, but also from culture to culture. Communities explore and transform these realities in many ways, through art, drama and narrative for example. In developing the coherence compiler we are particularly interested in the relationship between creativity and narrative as applied to education. Narrative offers us a way to play with the constraints of reality: to help learners to be creative. Used appropriately it also allows us to engage learners.

The narrative context of a learning episode has both cognitive and affective consequences. Incoherent or unclear narrative requires extra cognitive effort on the listener's part to disentangle the ambiguities. As a consequence the learner may be distracted from the main message of the learning episode, which may in turn detract from her ability to understand the concepts to be communicated. It may also disengage her altogether. On the other hand engaging narrative may motivate her to expend cognitive effort in understanding concepts to which she would not otherwise be inclined to attend. The Non-linear Interactive Narrative Framework identifies ways in which narrative might be exploited in interactive learning environments. The NINF distinguishes two key aspects of narrative:

Narrative guidance (NG): the design elements that teachers and/or software need to provide in order to help learners interpret the resources and experiences they are offered, and

Narrative construction (NC): the process through which learners discern and impose a structure on their learning experiences, making links and connections in a personally meaningful way.

\subsection{What is the Coherence Compiler?}

The Coherence Compiler is responsible for giving learners a coherent learning experience. The need for providing coherence is perhaps not very clear if you are imagining material drawn from a single source (say the 'Number Crew') as this content will already have 'coherence' designed into it; the content goes together, has a built in sequence with a clear structure contained in storylines and lesson plans that link video clips, worksheet and other activities in a coherent narrative (there is implicit narrative guidance). However, when we consider how we may wish to link diverse content, drawn from a variety of sources, into a unified learning experience the need for some means of maintaining coherence or supporting learners and their helpers in constructing this coherence becomes more evident (this may require more explicit narrative guidance). Somehow the Coherence Compiler needs to be able to generate or know about routes through appropriate (where appropriate means relevant to the learner's needs) content that make 'narrative' sense. The Coherence Compiler should also be able to guide learners and/or authors of learning experiences in 
creating their own coherent routes through potentially diverse and otherwise unrelated but relevant content, perhaps by providing access to suitable tools; e.g. search tools, guides that relate content to learning objectives, ways of relating past learning to current learning, etc. The issues raised here are common for a variety of schemes that wish to amalgamate materials from diverse sources into a coherent whole, see e.g. [10].

\subsection{How does the Coherence Compiler interact with other system components?}

In order to provide the kind of services suggested above the Coherence Compiler needs information about: the available content and its relation to other content; the learner's characteristics; the learner's history of activity; the learner's personal goals and curriculum goals; the tools available to help learners relate content, learning objectives and past learning; the tools available to help teachers build routes through content; and existing coherent routes through content (lesson plans, schemes of work, ways of identifying content that is part of a series, and popular associations between content).

Much of this information might be provided by the content management system or other system components: the content metadata, including relationship data; the Learner Model; logs of learner activity; a Curriculum or Pedagogic Model; a collection of suitable user interfaces (teacher / child / helper) for visualising content search results, learner activity and learning / curriculum objectives; a database of coherent trail information (e.g. lesson plans, other authored routes, popular routes, i.e. sequences of content that many similar learners have followed).

So, while the content management system and other components are able to successfully identify and retrieve content that is suited to a learner's needs and to present that content along with information about how it relates to other content elements, the value of the Coherence Compiler is that it enables the teacher and/or learner to create a coherent route through that suitable content. The Coherence Compiler provides user interfaces appropriate to each of its user groups (teacher / learners / learner collaborators, parents etc...) for those of its services, which are visible to users, i.e. tools for narrative construction and explicit narrative guidance..

\subsection{Coherence Compiler Interfaces Requirements}

Primarily for Teacher. The interface for teachers should: (i) Be able to assist primary teachers to find suitable content and make 'coherent paths' through it (e.g. lesson plans or schemes of work). (ii) Be capable of performing search on available content metadata in order to find content that suits the purpose of the author. (iii) Enable (and possibly encourage) teachers to add guidance and scaffolding elements to the lessons they create e.g. Reminders of the goal of the session, identification of subgoals and prompts to ask for help. (iv) Allow the teacher to reference content not known to the system but available to the teacher. (v) Allow the teacher to annotate links between content and activities and include instructions; e.g. (instructions and annotation in italic) - First watch section 1 of the video about elephant being max weight for the roller coaster \{ref clip and start and stop times\} think about the problem. How do you think the crew can work out which combinations of animals 
weigh less than the elephant? Now watch the solution ref clip\}. Now play the game \{ref interactive\} to help find combinations of animals that weigh less than the elephant. (vi) Allow the teacher to select level of system or learner control applicable to the session. (vii) Allow the teacher to select from amongst options for the nature and strength of the narrative guidance to be offered, see Figure 1.

Primarily for learners. The interface for learners should: (i) Provide access to the data and information that learners will need to construct their personal narrative understanding: i.e. learning history, available content, learning objectives, content menus and search facilities, etc. (ii) Remind learners of (macro and micro) objectives in a timely manner in order to focus their attention on a purposeful interpretation of the content. (iii) Guide learners towards accessing content that delivers these learning goals. Guidance may be more or less constraining depending on the learner's independence. (iv) Vary the degree of (system-user) narrative control over the sequence of events and activities or route through content, to match the needs of different learners. (v) Guide a child in choosing what to do next (for young children this guidance is likely to be very constraining - a linear progression of 'next' and 'back' buttons or a limited number of choices. For more independent learners guidance (and interface) would become less constraining. (vi) Enable the learner to record and reflect on their activity and progress towards goals. Possibly by annotating suitable representations of her activity log and objectives. Again, this needs to be done in a way that is intelligible and accessible to young children. (vii) Be able to suggest 'coherent paths' through content (to learners, parents, teachers) through analysis of content usage in authored paths and in other learners' activity histories. For example, if I choose to use a certain piece of video, and learners with similar profiles have used this perhaps what they chose to do next will also be suitable for me (something like the way Amazon suggests purchases?). Or if a piece of content I choose to incorporate in a 'lesson plan' has been used on other lesson plans maybe other pieces of content used to follow on from this content in those plans will be appropriate to the new plan. This feature will obviously become more useful over time as the system incorporates larger volumes of content and usage but will have to be careful not to confuse users with divergent recommendations. 


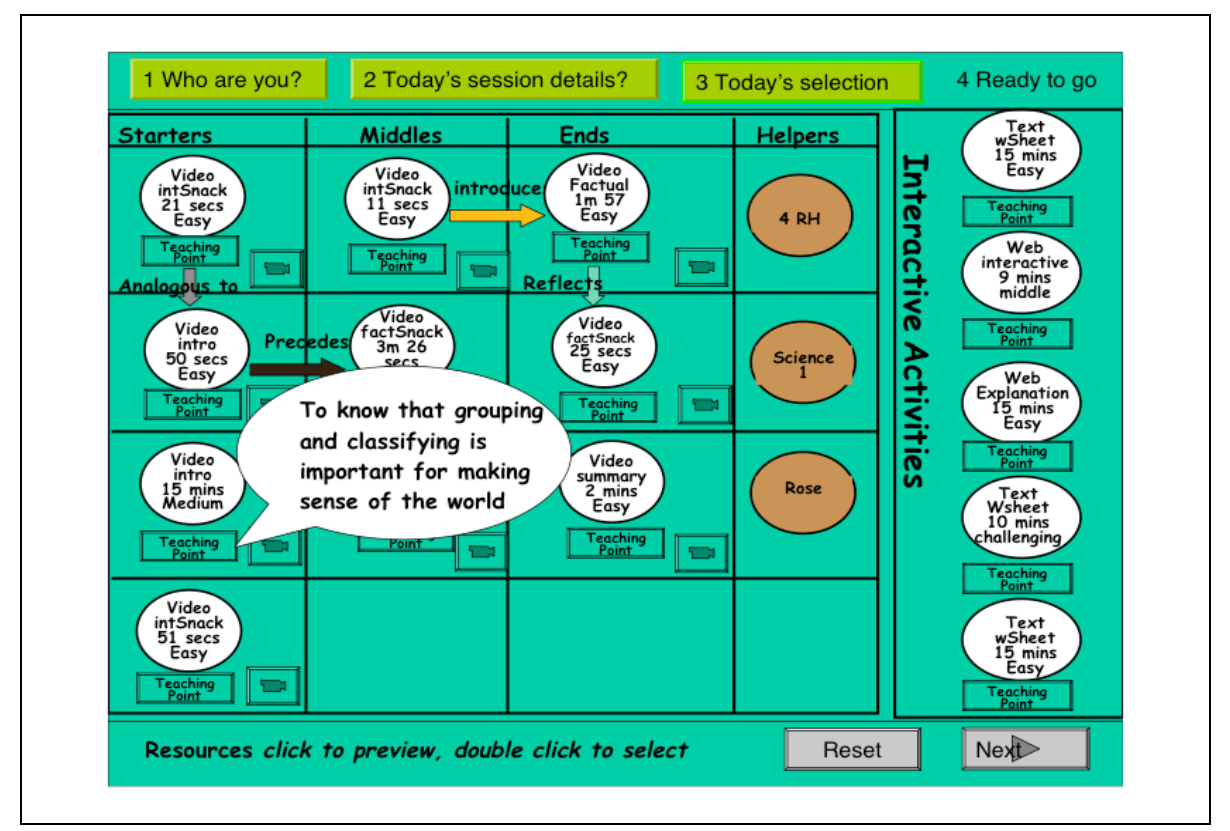

Figure 1. Mock up of interface for teachers

Primarily for Learners with Collaborators. The interface for learners with collaborators should allow learners (and their parents/guardian/teachers) to review and annotate the learner's history of interaction with the system. This could facilitate a form of collaborative parent child narrative construction. This interface might be a bit like a browser history, learners would be able to revisit past interactions. Maybe if asked what did you do today at school a child would be able to show as well as tell through the use of this feature. There are many challenging issues to address here including separating out individual and group learner models as well as assignment of credit.

Not Visible to users. Although not directly visible to users, the system should: (i) Have access to a record of a child's activity with the system. (ii) Have access to authored 'coherent journeys' through available content: coherent journeys are linked sequences of guidance comments, activities and content that make sense (e.g. existing lesson plans and schemes of work authored by material producers and/or users of the system, other sensible sequences of interaction and guidance possibly obtained through analysis of content usage by all learners). (iii) Be able to identify suitable content for a child's next interaction based on the record of her activity and the 'coherent journeys' described above. Decisions about suitable content will also involve consideration of the learner's individual identity and needs described in the learner model and pedagogic objectives (possibly described by the curriculum). (iv) Be able to choose/suggest 'paths' through content that are interesting/motivating to individual learners; i.e. if there are several paths through content/plans for learning at an appropriate level for this learner choose the one that is most likely to be interesting/motivating to this learner 


\section{Conclusions}

In this paper we have described the initial design of the Coherence Compiler for the HomeWork project. The HomeWork project is making existing content materials, including TV programs, available to learners. The original programs may not be used in their original entirety, but parts selected, re-ordered or repeated and interspersed with other materials and activities according to the needs of individual or groups of children. The Coherence Compiler is responsible for maintaining narrative coherence across these materials and across devices so that the learner experiences a wellordered sequence that supports her learning effectively. Such support may be provided both through narrative guidance and tools to support the learner's own personal narrative construction. Narrative guidance should be adaptive to the needs of the learner, it initially offers a strong 'storyline' explicitly linking new and old learning and then fades as the learner becomes more accomplished at making these links for herself. Such support may be provided both through narrative guidance and tools to support the learner's own personal narrative construction.

\section{References}

1. Luckin, R., Connolly, D., Plowman, L., and Airey, (2002) The Young Ones: the Implications of Media Convergence for Mobile Learning with Infants, in S. Anastopolou, M. Sharples \& G. Vavoula (Eds.) Proceedings of the European Workshop on Mobile and Contextual Learning, University of Birmingham, 7-11.

2. Luckin, R. and du Boulay, B. (2001) Imbedding AIED in ie-TV through Broadband User Modelling (BbUM). In Moore, J.D., Redfield, C.L. and Johnson, W.L. (Eds.) Artificial Intelligence in Education: AI-ED in the Wired and Wireless Future, Amsterdam: IOS Press, 322--333.

3. Luckin, R. and du Boulay, B. (1999) Capability, potential and collaborative assistance, in J. Kay (Ed) UM99 User Modelling: $7^{\text {th }}$ International conference on user modeling, Banff, Alberta, Canada, CISM Courses and Lectures, No. 407, Springer-Verlag, Wien, 139-148.

4. Luckin, T. and Hammerton, L. (2002) Getting to Know me: Helping Learners Understand their Own Learning Needs through Metacognitive Scaffolding, in S.A. Cerri, G. Gouarderes \& F. Paranguaca (Eds), Intelligent Tutoring Systems, Berlin: Springer-Verlag, 759-771.

5. Luckin, R., Plowman, L. Laurillard, D, Stratfold, M. and Taylor, J. (1998) Scaffolding Learners' Constructions of narrative in A. Bruckerman, M. Guzdial, J. Kolodner and A Ram (Eds) International Conference of the Learning Sciences, Atlanta: AACE, 181-187.

6. Plowman, L., Luckin, R., Laurillard, D., Stratfold, M., \& Taylor, J. (1999). Designing Multimedia for Learning: Narrative Guidance and Narrative Construction, in the proceedings of CHI 99 (pp. 310-317). May 15-20, 1999, Pittsburgh, PA USA.: ACM.

7. Bruner, J. (1996). The Culture of Education. Harvard University Press, Cambridge MA.

8. Vygotsky, L. S. (1986). Thought and Language. Cambridge, Mass: The MIT Press.

9. Boden, M. A. (2003) The Creative Mind: Myths and Mechanisms. London, Weidenfeld and Nicolson.

10. AFAIDL Distance Learning Initiatve: www.cbd-net.com/index.php/search/show/536227 